\section{Eliminate}

\section{misundertanding}

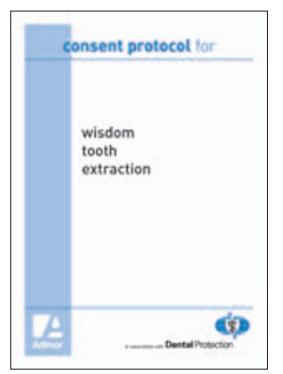

Admor, in association with Dental Protection, offer a unique range of practical treatment consent forms. These forms have been designed to help both dentists and patients establish consent before treatment commences, helping to eliminate any future misunderstandings.

Presented in pad format, a new document can be generated for each patient. The pages provide general instructions for the dentist to ensure consent is gained in an appropriate manner, and general information for patients explaining why the procedure is necessary at all, along with information on the upcoming procedure, advice before and after treatment and consultation record patient and dentist copies.

The forms are intended to provide the dentist with a checklist of issues that need to be discussed, provide patients with pre- and post-treatment advice, and also to provide a record of the patient's agreement and understanding.

Reader response number 51

\section{Magnetised unit}

The Hydrofloss Kitty Waterjet is effective in helping patients remove bacteria that brushing and flossing cannot. The new Hydrofloss unit has had a magnetic device added to it, making it twice as effective as an unmagnetised irrigator.

There are now a number of ways that you and your patients can benefit from the Hydrofloss Kitty Waterjet, including having the unit shipped direct to your patient, as well as incentives where you can accrue credits for stock each time one of your patients orders a unit through the company's coded order system.

Reader response number 53

\section{Maximum adhesion and aesthetics}

Maxcem from Kerr is a new self-etch, self-adhering resin cement that provides good bond and mechanical strengths and aesthetics with simplicity. A two-part paste and dual-cure resin cement, Maxcem's system allows all the essential ingredients to etch, prime, bond and cement to be combined into one product.

Maxcem features exceptional material stability, direct dispensing with automix syringe, a highly efficient dark-cure mechanism and long-term colour stability in both uncured and cured states.

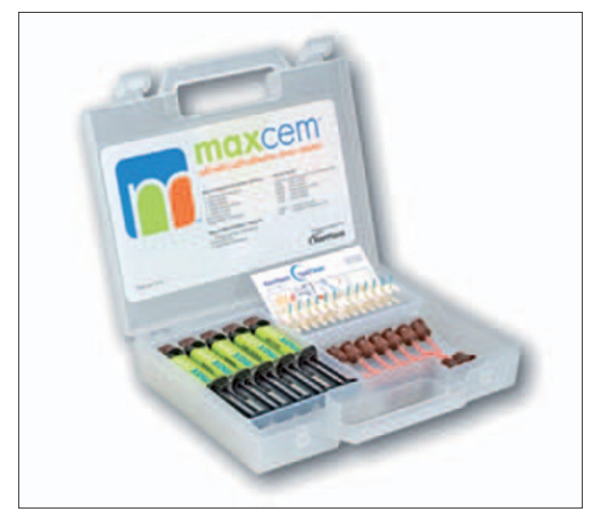

It can be used for indirect cementation of crowns, bridges, inlays, onlays and posts.

Reader response number 50

\title{
New resorbable membrane
}

Ossix Plus porcine collagen resorbable membrane allows predictable outcome and ease of use in guided bone regeneration and treatment of peri-implant defects. It can be cut to the size and shape required and is easy to place, without the need for tacking or suturing the material in place. It can effectively be applied to a variety of common guided bone regeneration defects.

The product, which is derived from porcine collagen, uses glymatrix linking technology and is flexible and adaptable for varying bone topographies. Its

\section{Tailor made art}

Designer Dental has announced the addition of a new dimension to their poster, print and canvas portfolio. The new prints are exclusive to Designer Dental and have been taken from original photographs by dentist Chris May. ability to function as a barrier over four to six months allows sufficient time for osseous defects to achieve optimum bone regeneration and the membrane maintains its porosity to permit the passage of fluids and nutrients related to soft tissue health.

At the same time, Ossix Plus does not have to be removed where exposed. It maintains its barrier function whilst simultaneously encouraging soft tissue to naturally epithelialise over the exposure. It is available from 3i Biomet.

Reader response number 52

These prints do not have a dental theme; they are purely decorative and pleasing to look at and can be purchased individually or in matching pairs. They come in three standard sizes, or if you prefer, can be tailor-made to any size you wish.

Reader response number 54 

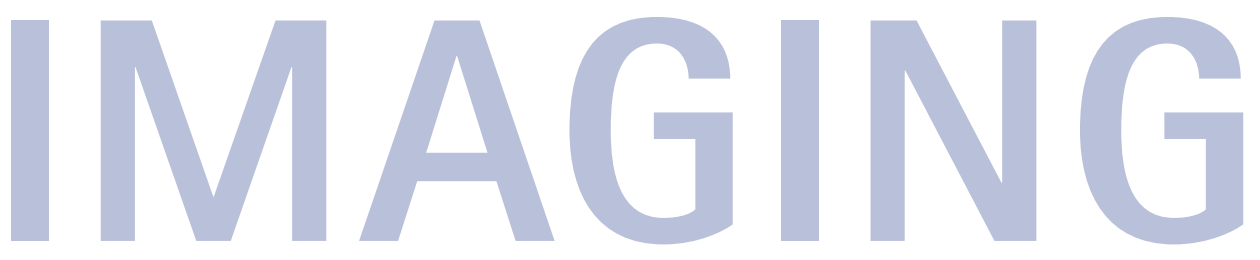

\section{Panoramic imaging}

The Planmeca ProMax product line provides the dental practice with a large selection of panoramic and tomographic imaging programs including $3 \mathrm{D}$ and cephelostat.

Each Planmeca ProMax incorporates a digitally controlled X-ray generator and an ethernet-based data path to its digital sensor for high capacity of image transfer.

To achieve this wide selection of image programmes, the Planmeca ProMax utilises the unique three joint SCARA arm. Combined with the image geometry software the ProMax can produce any movement pattern that is required in existing or future exposure programs; hence imaging capabilities have no mechanical restrictions. Moreover, this technology allows upgrade of new imaging programs through software and enables optimal radiography for each individual patient type and for virtually any need of maxillofacial diagnostics.

\section{Reader response number 56}

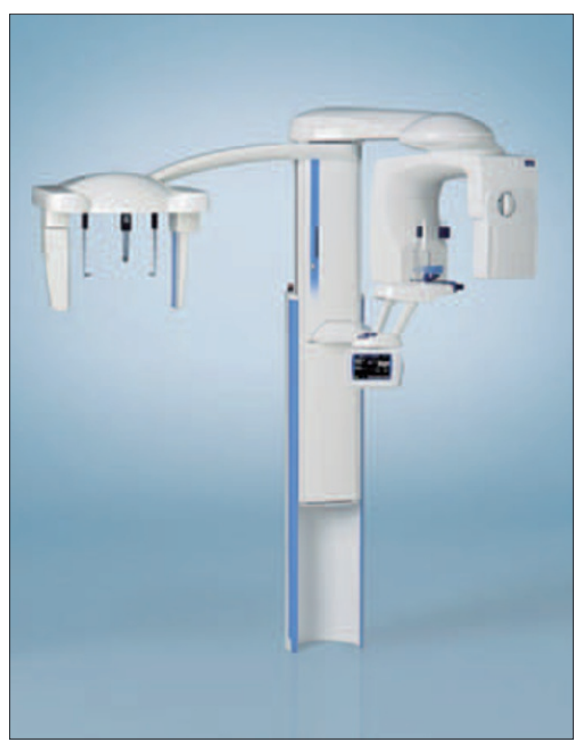

\section{Multi-function X-ray machine}

The high frequency technology of the new Phot-X II multi-function DC Xray machine decreases the soft X-ray absorbed to the patient's tissue. The tube voltage, current and exposure time can all be selected according to the patient, which helps to avoid over-exposure to radiation.

The Phot-X II offers a minimum exposure time of 0.01 seconds and is compatible with any type of digital imaging system. The pre-programmed timer is selectable for digital systems and two different types of films. A total of 16 film speeds are available.

The lightweight, compact, easy to hold tube head enables the operator to align the tube accurately for reproducible, high contrast, crisp radiographs with excellent image quality. The focal spot is set central to the head allow-

\section{One shot radiography}

A new dual-purpose digital OPG/Ceph machine has been added to Schick Technologies digital imaging system range. Called the Schick CDRPanX-C, it enables all the standard cephalometric images to be taken with just one shot, as well as, like its sister OPG machine the Schick CDRPanX, a full range of pan, segmented pan, TMJ and maxillary sinus images.

Also part of the range is the iPan retrofit kit which converts most film-based OPG machines to digital; the CDR cabled sensor and CDR Wireless cable-free sensor, both for intraoral radiography; the CDR SDX X-ray generator, designed especially for use with the company's intraoral sensors and digital intraoral radiography in general; and finally

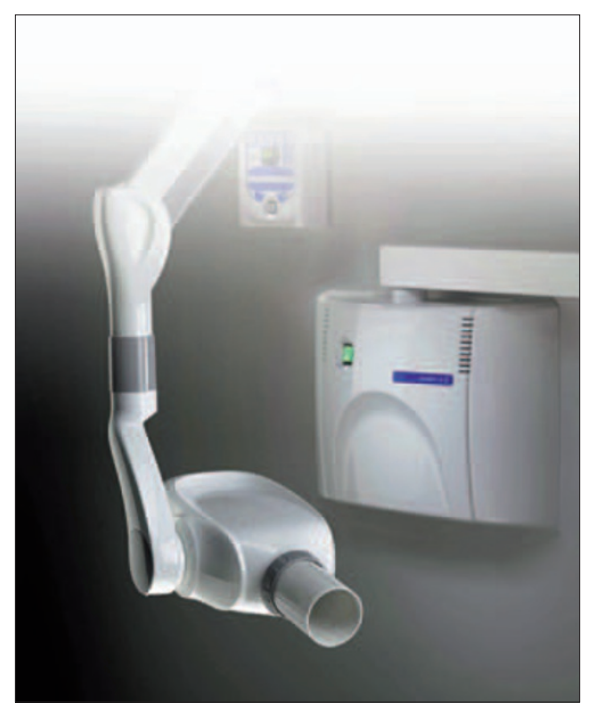

ing for a shorter cone to be used. The Phot-X II is available in four types of installations: wall-mounted; room type with stool; mobile type with castors; and floor-mounted.

Reader response number 55

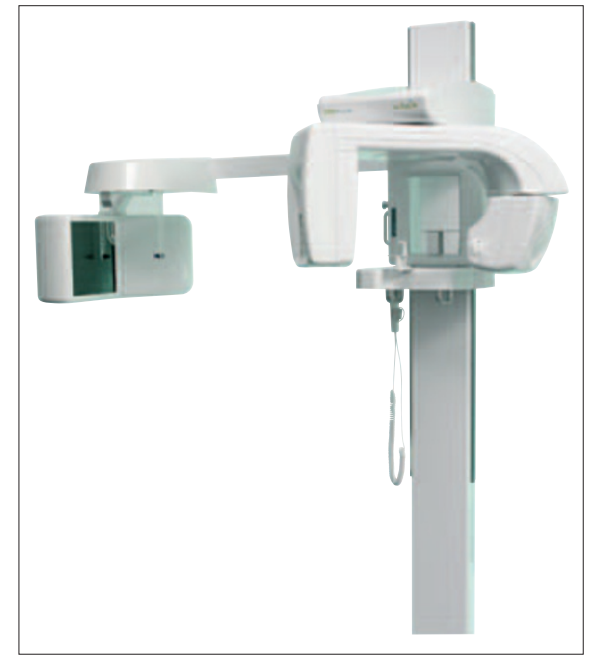

the 'plug and play' Schick USBCam intraoral camera.

Reader response number 57 


\section{Free imaging advice}

Imaging Technologies supply fully digital intraoral cameras from leading manufacturers. For example, the Real Hi T from Owandy offers true to life images by way of five LEDs evenly distributed

\section{Durable mounts}

EZ view masked mounts from Dentsply Rinn are an accurate and simple way to view your patient's history of dentition, disease progression or healing. Due to the durable, non-glare material that they are made from, the film is protected from scratches, keeping them safe.

Duplications with exceptional clarity can be made without removal from the mount and the patient details will appear on the duplicate. Due to the top-entry pockets, the film can be quickly placed, saving time, and as they are ultra-thin,

\section{High magnification}

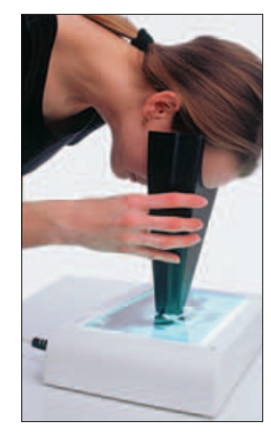

The SDI Directa Xray magnifier from Trycare Dental Linkline enables dentists to view radiographs more clearly and easily. A prerequisite for the accurate interpretation of X-ray images is the elimination of background

\section{Hygienic sleeves}

Kerr has announced the distribution of a complete range of X-ray film holders with a new universal design. The new design is robust yet slim in appearance and is compatible with all films and imaging plates up to $2 \mathrm{~mm}$ thickness.

\section{No over-exposure}

The DMDS Suni digital X-ray system is the latest in digital imaging, providing you with ultra-thin sensors for even more accurate images at the very highest resolution. Being extremely thin, the sensors are patient friendly and also dentist friendly, as they are provided in familiar sizes similar to traditional X-ray films.

Normal X-rays provide you with 15 line pairs whereas the DMDS digital X-ray system's MegaPixel Dentistry provides you with 22 line pairs and over 2 million pixels, which is $45 \%$ higher than films. around the lens that flood the buccal cavity with light. The auto-focus discreetly ensures optimal distance setting, whilst the image, which is digitised at source via a high-tech CCD sensor, reproduces every detail in natural colour.

Reader response number 58

they can be easily stored. Email sales. weybridge@dentsply-gb.com before 31 July 2007 for your free sample.

Reader response number 59

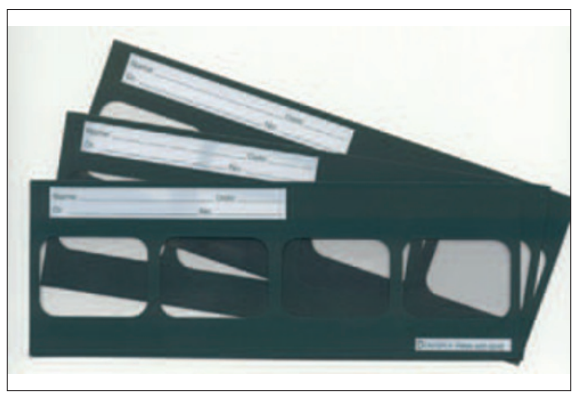

light interference so that fine detail is more sharply contrasted.

The matt black inner surface of the SDI Directa X-ray magnifier absorbs stray light, thus increasing contrast. Designed to accommodate the wearing of spectacles, at twice the magnification and with minimal light pollution the SDI Directa X-ray magnifier makes it easy to inspect the fine detail in X-rays. This allows viewing in almost dark room-like conditions and at twice the size.

Reader response number 60

The holders withstand cold disinfection, thermo-disinfection and steam sterilisation at $134^{\circ} \mathrm{C}$ for up to 50 cycles.

The clamping mechanism allows the use of hygienic sleeves without jeopardising the photostimulable layer and with minimal encumbrance.

Reader response number 61

There will not be any re-takes as the film cannot be overor under-exposed, allowing absolutely precise instantimages every time. You will be able to view the images immediately on your screen with no time wasting, and with 90\% less radiation produced, your patients are also likely appreciate this technology.

Reader response number 62 